\title{
Tipos de Problemas que Provocan la Generación de Argumentos Inductivos, Abductivos y Deductivos
}

\author{
Types of Problems that Elicit Inductive, Abductive, and Deductive \\ Arguments
}

\author{
Oscar Molina* \\ ORCID iD 0000-0003-4797-1173 \\ Carmen Samper** \\ ORCID iD 0000-0003-2342-8950
}

\begin{abstract}
Resumen
En este artículo se presenta una relación entre tipos de problemas abiertos de conjeturación en geometría, preferiblemente abordados en Entornos de Geometría Dinámica (EGD), y clases de argumentos producidos. Se expone cómo cada tipo de problema provoca la producción de argumentos (inductivos, abductivos o deductivos) durante el proceso de resolución. Para apoyar la idea, se exponen estrategias de solución producidas por estudiantes de un curso de geometría plana de un programa de formación inicial de profesores de matemáticas (Universidad Pedagógica Nacional, Colombia), cuando abordan problemas que involucran el objeto mediatriz de un segmento. Usando el modelo de argumento propuesto por Toulmin para analizar dichas estrategias, se identifican los tipos de argumentos asociados a cada tipo de problema. Se señala cómo la tipología de problemas puede contribuir al conocimiento didáctico-matemático del profesor de matemáticas.
\end{abstract}

Palabras clave: Tipos de Problemas Abiertos de Investigación. Argumento Inductivo. Argumento Abductivo. Argumento Deductivo. Entorno de Geometría Dinámica.

\begin{abstract}
This paper presents a relation between the types of conjecturing open problems in geometry, preferably solved using Dynamic Geometry Systems, and the types of arguments produced. The aim of this paper is to show how each type of problem can elicit the production of certain types of arguments (inductive, abductive, and deductive) during the problem-solving process. To back this idea, we use examples of specific problems (that involve the geometric object perpendicular bisector of a segment) and show solution strategies produced by students of a plane geometry course included in a pre-service mathematics teacher program (Universidad Pedagógica Nacional, Colombia). Using the argument model proposed by Toulmin to analyze these strategies, we identify the types of arguments associated with each type of problem. Finally, we indicate the typology of problems that can contribute to the didactic-mathematical knowledge of the mathematics teacher.
\end{abstract}

Keywords: Types of Conjecturing Open Problems. Inductive Argument. Abductive Argument. Deductive Argument. Dynamic Geometry System.

\footnotetext{
* Doctor en Educación Matemática, Universidad de Los Lagos (ULAGOS). Profesor de Planta, Universidad Pedagógica Nacional (UPN), Bogotá, Colombia. Dirección postal: Calle 182 N 45-24 Apartamento 1901 Torre 3, Bogotá, Colombia, C.P: 111166. E-mail: ojmolina@pedagogica.edu.co.

** M.A. en Matemáticas, University of Maryland (U.M.D.). Profesora Emérita, Universidad Pedagógica Nacional (UPN), Bogotá, Colombia. Dirección postal: Tv. 2A N ${ }^{\circ}$ 67-71, Apartamento 702, Bogotá, Colombia, C.P: 110231. E-mail: csamper@ pedagogica.edu.co.
} 


\section{Presentación}

Actualmente, una tendencia en el ámbito educativo es recomendar que los currículos y las prácticas de instrucción se sustenten en ideas del campo Resolución de Problemas (LESH; ZAWOJEWSKI, 2007; ONUCHIC; ALLEVATO， 2011; SANTOS-TRIGO， 2014; ZIMMERMANN, 2016).

Esta perspectiva reconoce la resolución (y planteamiento) de problemas como aspecto central para el desarrollo del pensamiento matemático de los estudiantes, pues les posibilita idear estrategias, usar recursos y desarrollar disposición para realizar actividades que involucran el quehacer matemático. Tal como dice Schoenfeld (1992), la resolución de problemas posibilita un escenario que no solo se focaliza en cálculos y deducciones, sino que involucra la observación de patrones, y la formulación, testeo y demostración de conjeturas.

Schoenfeld (1992) plantea como reto para la instrucción matemática basada en la resolución de problemas crear condiciones para generar un ambiente que favorezca las acciones propias de la actividad matemática. Este contexto implica reflexionar sobre los cambios que demanda la forma de presentar los contenidos en el aula de matemáticas, si la resolución de problemas propicia la actividad matemática de los estudiantes, y si el profesor realmente asume esta perspectiva en su práctica.

Con respecto a lo último, se ha mostrado que los profesores no necesariamente incluyen en su práctica tal actividad, posiblemente porque en su formación inicial tuvieron poca experiencia como resolutores, o porque no tuvieron suficientes oportunidades para reflexionar sobre el potencial didáctico-pedagógico de esta perspectiva (FELMER et al., 2015; ZIMMERMANN, 2016).

En las últimas tres décadas ha existido el interés por involucrar la argumentación en los currículos escolares como medio para desarrollar competencia matemática de los estudiantes (BALL; BASS, 2003; YACKEL; HANNA, 2003; MARIOTTI, 2006). Ello porque, al igual que sucede con la resolución de problemas, propicia acciones propias del hacer matemáticas como inducir o abducir propiedades de hechos empíricos para formular conjeturas, proveer pruebas deductivas formales de hechos previamente conjeturados para validarlos, y comunicar los resultados obtenidos. Ahora bien, favorecer las prácticas argumentativas en el aula exige que los profesores pongan en juego tanto sus conocimientos y prácticas sobre diversos tipos de argumentos como los relativos a aspectos didácticos (o sus propias creencias), pues ello les ayuda a seleccionar tareas de aprendizaje que promuevan la argumentación y prever (y luego evaluar) la actividad de los estudiantes (LIN et al., 2012). 
En este sentido, saber sobre diferentes tipos de argumentos y reconocer la diferencia entre los empíricos (por ejemplo, inductivos y abductivos) y los formales (deductivos) debe ser parte del conocimiento de un profesor de matemáticas. Más aún, conocer diferentes tipos de tareas relativas a la argumentación y cómo abordarlas, y reconocer la relación entre estas y la actividad matemática que ello implica debe ser otro de los elementos de dicho conocimiento del profesor (STYLIANIDES; BALL, 2008).

Con el fin de vincular los dos aspectos citados, la resolución de problemas y la argumentación, en un programa de formación inicial de profesores, desde el año 2005 hemos participado en una innovación - liderada por el grupo de investigación Aprendizaje y Enseñanza la Geometría - $\mathcal{A} \bullet \mathcal{G}$ - de la Universidad Pedagógica Nacional - UPN - (Colombia) - que busca generar un entorno favorable para aprender a demostrar en cursos de geometría (CAMARGO et al., 2009; PERRY et al., 2012).

El esfuerzo didáctico de tal innovación recae sobre los siguientes elementos: $i$ ) problemas abiertos de conjeturación; ii) el uso de un Entorno de Geometría Dinámica (EGD) como artefacto que posibilita la exploración de situaciones y en consecuencia, soluciones a los problemas propuestos; y iii) la interacción social en la clase, ya sea entre estudiantes, cuando resuelven conjuntamente problemas, o entre profesor y estudiantes, cuando ellos exponen públicamente su solución a los problemas y argumentan las ideas que tienen relacionadas con su solución.

Este artículo presenta parte de los resultados obtenidos de un proyecto de investigación ${ }^{1}$ desarrollado en 2014 por el grupo $\mathscr{A} \bullet \mathcal{G}$. Específicamente, se expone la relación entre la tipología de problemas (para ser abordados en un Entorno de Geometría Dinámica, EGD) propuesta por el grupo, y una tipología de argumentos (inductivos, abductivos, deductivos) basada en el Modelo de Toulmin para un argumento (BOERO et al., 2010). Para ilustrar la relación, se usan hechos ocurridos en clases de geometría plana del programa de formación de profesores de la universidad antes citada.

Con este trabajo pretendemos contribuir al desarrollo curricular de los programas de formación de profesores de matemáticas, particularmente en lo que respecta a los elementos del conocimiento del profesor citados por Stylianides y Ball (2008) y presentados anteriormente. Es necesario que los futuros profesores conozcan las matemáticas de tal

\footnotetext{
${ }^{1}$ Proyecto denominado Conjeturas y organización del contenido matemático en clase, desarrollado en 2013 2014 y financiado por el Centro de Investigación de la Universidad Pedagógica Nacional y Departamento Administrativo de Ciencia, Tecnología e Innovación de Colombia (Colciencias).
} 
manera que sean útiles para la enseñanza - no es suficiente que sepan las matemáticas que ellos van a enseñar (BALL; LUBIENSKI; MEWBORN, 2001).

Por ello, es deseable que en los programas de formación de profesores se ofrezcan experiencias en donde ellos no solo conozcan los tipos de argumentos, sino que accedan a ellos de tal forma que evidencien su utilidad para la enseñanza. Una manera para lograrlo es que ellos experimenten, en su formación, diferentes tipos de tareas que favorezcan su aprendizaje de la argumentación mediante su abordaje y resolución, y que además le ayuden a ver cómo este proceso matemático se relaciona con la enseñanza, vía los tipos de tareas que se proponen (STYLIANIDES; STYLIANIDES, 2006); en otras palabras, conciban la resolución de ciertos tipos de problemas como un camino para involucrar la actividad argumentativa en la enseñanza y tengan herramientas para reconocer las características de ese tipo de actividad en el proceso de enseñanza.

En este sentido, sugerimos tipos de problemas abiertos de conjeturación que, al ser empleados con futuros profesores en su proceso de formación, abren un espacio para que ellos puedan ganar experiencia como resolutores $\mathrm{y}$, en ese marco, fortalezcan su competencia argumentativa. De manera concreta, presentamos cómo cada tipo de problema, caracterizado por la estructura de su enunciado, favorece un tipo específico de argumento.

Vale indicar que varios investigadores han mostrado cómo problemas abiertos resueltos en EGD favorecen procesos argumentativos (e.g., ARZARELLO et al., 2012; BACCAGLINI-FRANK; MARIOTTI, 2010; PEDEMONTE, 2007). Sin embargo, no explicitan cómo la estructura del enunciado del problema puede favorecer la producción de diferentes tipos de argumentos, claves para la elaboración de conjeturas y, eventualmente, la producción de su demostración.

Siguiendo la idea de Stylianides y Stylianides (2006), los problemas que proponemos, tipificados según su estructura y su relación con tipos de argumentos, además de permitir que los profesores en formación se involucren en procesos de argumentación y resolución de problemas, les provee, también, una herramienta concreta (los tipos de problemas) a través de las cuales estos procesos matemáticos pueden ser enseñados cuando ejerzan como profesores.

El texto se estructura como sigue: primero se presentan referentes teóricos; luego, se exponen los aspectos metodológicos seguidos para el análisis. Posteriormente, se describe los tipos de problemas, su relación con los tipos de argumentos y se ilustra con ejemplos cada uno de los casos. Para concluir, se exponen unas reflexiones finales.

\section{Marco conceptual}


En esta sección se presenta, en primera instancia, lo que se concibe como problema abierto de conjeturación. Se expone qué es un argumento y se describen tipos de argumentos empleando el Modelo de Toulmin según la postura de Boero et al. (2010). Se presentan, de manera breve, referentes que relacionan los procesos de argumentación, conjeturación y demostración, y perspectivas que aluden a los EGD y su potencial en los procesos de resolución de problemas y de argumentación.

\subsection{Problema y problema abierto de conjeturación}

Asumimos un problema abierto como aquel en el que el enunciado no revela su solución o respuesta. Cuando un problema pide, explícitamente, establecer una conjetura (i.e., proposición condicional $p \rightarrow q$ ) que exprese relaciones de dependencia entre elementos o propiedades de las figuras (o configuraciones) involucradas en la situación, este se denomina problema abierto de conjeturación (BACCAGLINI-FRANK; MARIOTTI, 2010).

Específicamente, la innovación descrita en la sección anterior pretende que los problemas que se proponen en clase sean lo que Ponte, Fonseca y Brunheira (1999) denominan investigaciones matemáticas. En primer lugar, estas son problemas abiertos que permiten a los resolutores diversas alternativas de exploración y de solución, dado que los datos o meta implican un grado de indeterminación significativo (PONTE, 2004).

En segundo, son tareas que pretenden que el resolutor efectúe un proceso semejante al que realiza un investigador matemático, es decir, que determine la cuestión que se va a estudiar, ponga a prueba y refine conjeturas, explique o demuestre, y comunique resultados. Finalmente, son tareas que dan lugar a la introducción de un nuevo objeto de estudio para discutir y producir su significado, o hacer que un objeto conocido se resignifique (PONTE, 2003). Como se ilustrará, los tipos de problemas abiertos que se presentarán cumplen con las características descritas.

\subsection{Argumentos y argumentación}

Según el modelo básico de Toulmin (BOERO et al., 2010), un argumento es un enunciado oral o escrito, de estructura ternaria, que relaciona proposiciones particulares (datos y aserción) y una general (garantía). En cualquier argumento, inicialmente se expresa un punto de vista (una afirmación, una opinión), denominado aserción (q) (claim en términos Toulminianos); luego se proporcionan datos ( $p$ ) (o data) para apoyar la aserción. La garantía 
(r) (o warrant) justifica el uso de los datos como soporte para la aserción.

La garantía, que puede ser expresada como un principio o una regla, actúa como un puente entre los datos y la aserción. Las proposiciones pueden no ser explícitas, pero para formalizar lo expresado debe ser posible identificarlas. La forma como se relacionan las proposiciones particulares ( $p$ y $q$ ) y la general $(r)$ define el tipo de argumento: deductivo, inductivo o abductivo. La siguiente descripción ${ }^{2}$ para cada tipo de argumento es propuesta por el grupo $\mathscr{A} \bullet \mathcal{G}$ y presentada en (PERRY et al., 2013).

En un argumento deductivo se aplica una proposición general conocida $(r: p \rightarrow q)$ a unos datos que se tienen $\left(p_{1}\right.$ : particularización de $p$ ), para obtener necesariamente la aserción $\left(q_{1}\right.$ : particularización de $\left.q\right)$. El esquema del argumento es: $\left(p_{1} \wedge r\right) \rightarrow q_{1}$ (Diagrama $\left.1^{3}\right)$. Este tipo de argumentos ocurre, primordialmente, en el proceso de demostración de una conjetura (Figura 1).

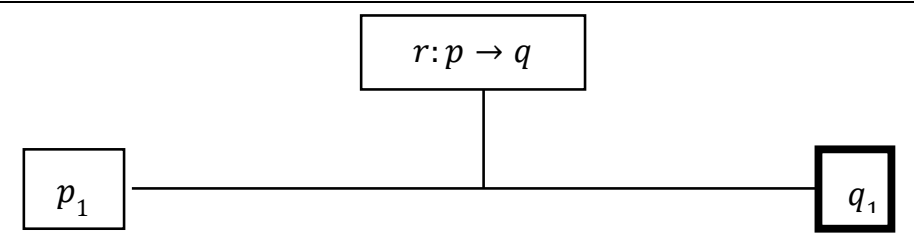

Figura 1 - Diagrama que esboza lo inferido en un argumento deductivo Fuente: elaborada por los autores

En un argumento inductivo se tienen $n$ casos que particularizan la proposición $p\left(p_{1}\right.$, $\left.p_{2}, p_{3}, \ldots, p_{n}\right)$ para los cuales, para cada uno, se satisface la proposición general $(q)$. Se concluye la proposición general $(r: p \rightarrow q)$. El esquema del argumento es: $\left(p_{1} \wedge q\right) \wedge\left(p_{2} \wedge\right.$ q) $\wedge\left(p_{3} \wedge q\right) \wedge \ldots\left(p_{n} \wedge q\right) \rightarrow r($ Figura 2$)$.

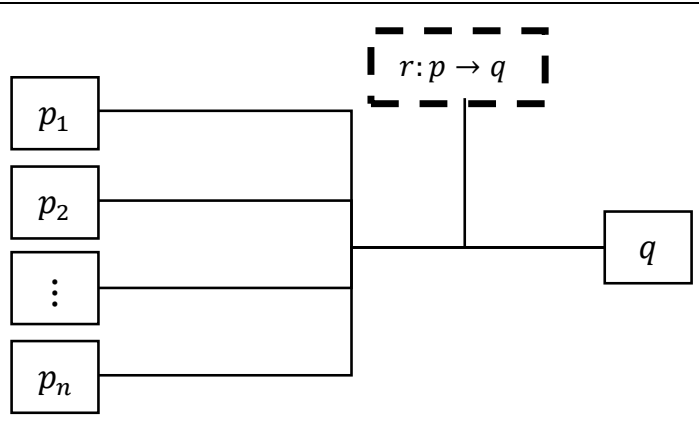

Figura 2 - Diagrama que esboza lo inferido en un argumento inductivo Fuente: elaborada por los autores

La conclusión obtenida es de índole provisional, es una conclusión plausible y para

\footnotetext{
${ }^{2}$ Cuando se presenten los tipos de problemas, se ejemplificará cada tipo de argumento.

${ }^{3}$ Se presenta, en un cuadro en negrilla, lo que se infiere en cada tipo de argumento.
} 
indicarlo en el diagrama usamos una línea punteada. Sería una conclusión válida si $q$ coexiste con todos los casos posibles de $p$, situación que se podría esquematizar con la tautología: $(p \wedge q) \rightarrow(p \rightarrow q)$. Si es posible validarla, se constituye en un nuevo elemento del sistema teórico. Los argumentos inductivos se dan, principalmente, en el proceso de conjeturación.

En un argumento abductivo, la proposición particular que se tiene se refiere a un hecho que se observa $q_{1}$ (caso de $q$ ). Se conocen proposiciones generales $r_{1}: p \rightarrow q, r_{2}: s \rightarrow$ $q, \ldots$ y, por ejemplo, se concluye que es posible el hecho $p_{1}$ (caso de $p$ ). El esquema del argumento es $\left(q_{1} \wedge r\right) \rightarrow p_{1}$ (Diagrama 3). La conclusión (o inferencia) así obtenida es de índole provisional y plausible; indicamos esto en el esquema con el símbolo “- $\rightarrow$ ”.

Cabe mencionar que la procedencia de la regla general no es única: o es una regla hipotética que proviene de una exploración empírica o es una regla aceptada como elemento del sistema teórico con el que se cuenta, elegida en una exploración teórica. Si bien en ambos casos $p$ es posiblemente verdadero, en el primero está el asunto de la validez de la regla, cosa que podría desencadenar en una argumentación deductiva que permita justificarla. Los argumentos abductivos pueden surgir en los procesos de conjeturación y de demostración de una conjetura (Figura 3).

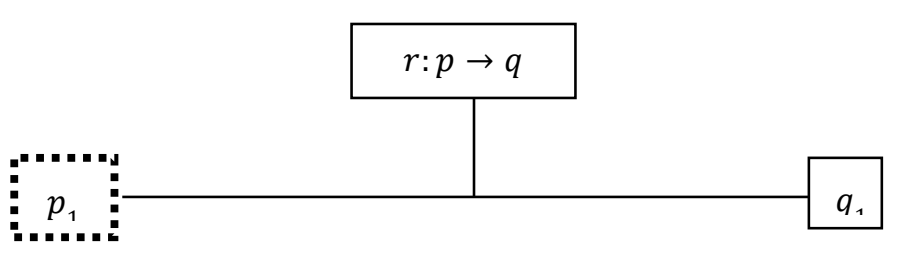

Figura 3 - Diagrama que esboza lo inferido en un argumento abductivo Fuente: elaborada por los autores

Acorde con las descripciones previas de argumento, entendemos por argumentación al acto comunicativo en el que se provee la formulación de argumentos para apoyar una idea.

\subsection{Entornos de Geometría Dinámica - EGD}

Como los tipos de problemas que se describirán requieren el uso de geometría dinámica, se presenta un marco referencial que muestre la relación entre los EGD y los procesos de resolución de problemas, de conjeturación y de argumentación. Baccaglini-Frank, Mariotti y Antonini (2009) afirman que los EGD han revolucionado el enfoque para entender la compleja relación entre representaciones gráficas y conceptos en la geometría euclidiana. En particular, mejoran el razonamiento geométrico en la resolución de problemas, pues promueven la exploración visual y el descubrimiento. 
En un EGD, las figuras representadas a través de una secuencia de comandos elegidos por el usuario son configuraciones con una lógica que se corresponde, en esencia, a los postulados de la geometría euclidiana. Las propiedades geométricas que obedecen los comandos controlan la aparición de invariantes bajo la función de arrastre. Por ello, la dependencia lógica intrínseca entre las propiedades de los elementos de la figura dinámica puede afectar la interacción entre los componentes figurales y conceptuales implicados en la solución de una tarea (LABORDE, 2000).

Específicamente, algunos investigadores han mostrado cómo los diferentes tipos de arrastre y la posibilidad de ver, en tiempo real, muchos casos de una situación, favorece la producción de argumentos de diverso tipos (inductivos, abductivos) cuando abordan un problema (e.g., ARZARELLO et al., 2002; BACCAGLINI-FRANK; MARIOTTI, 2010; STYLIANIDES; STYLIANIDES, 2005); provoca la construcción de conjeturas y su demostración (producto de la explicitación de propiedades dependientes de otras), y hace ostensivas las estrategias llevadas a cabo por los estudiantes (e.g., CAMARGO et al., 2009; SAMPER et al., 2010; SANTOS-TRIGO, 2014).

Presentamos, grosso modo, una descripción de los diferentes tipos de arrastre propuestos por Arzarello et al. (2002) que se tendrán en cuenta para hacer el análisis de las producciones de los estudiantes: arrastre libre, consistente en mover los puntos base de la construcción aleatoriamente; arrastre limitado/ligado, consistente en mover un punto sólo sobre el objeto al que pertenece; arrastre guiado, consistente en mover los puntos base de la representación para darle una forma determinada; arrastre de lugar ficticio, consistente en mover un punto base para que el dibujo conserve una propiedad descubierta (el punto que se mueve sigue un camino sin que los usuarios se den cuenta de ello); arrastre mantenido (BACCAGLINI-FRANK; MARIOTTI, 2010), consistente en mover un punto base para que el dibujo conserve una propiedad descubierta o deseada (el punto que se mueve sigue un camino que los usuarios perciben y que al poner traza al punto evidencian).

\section{Aspectos metodológicos}

Los datos del presente reporte hacen parte de un estudio investigativo más amplio, llevado a cabo por el grupo $\mathscr{A} \bullet \mathcal{G}$ durante 2013-2014. El propósito de este fue determinar elementos de la clase, relativos a las decisiones del profesor y a la participación de los estudiantes, que intervienen para organizar y desarrollar el contenido de un curso de 
geometría plana, a partir de las conjeturas formuladas por los estudiantes, producto de la solución de problemas.

El análisis de los episodios de clase, en dicho estudio, se realizó mediante la estrategia metodológica experimento de enseñanza (STEFFE; THOMPSON, 2000). En tal sentido, fue diseñada e implementada una secuencia de enseñanza y posteriormente analizada dicha implementación. En lo que sigue, presentamos una descripción de la población y del contexto del estudio, de la recolección de los datos (donde se caracteriza la secuencia objeto de este reporte) y cómo los autores de este artículo hicieron el análisis preliminar (de la potencial actividad de los estudiantes) y retrospectivo (de la actividad llevada a cabo por los estudiantes) para precisar la relación entre los tipos de problemas (caracterizados por la estructura de su enunciado) y el tipo de argumento (inductivo o abductivo) que provocan. Se precisan, además, las herramientas analíticas empleadas para ello.

\subsection{Contexto y población del estudio}

El contexto experimental del estudio fue el curso de geometría euclidiana plana, ubicado en el segundo semestre del programa de formación inicial de profesores de matemáticas de la Universidad Pedagógica Nacional (Colombia). Se consideraron dos grupos de dicho curso, uno realizado en el primer semestre del año 2013 (diecisiete estudiantes), y el otro desarrollado en el segundo semestre del mismo año (catorce estudiantes); cada curso fue dirigido por cada autor de este reporte, respectivamente. Las edades de los estudiantes oscilaban entre 18 y 24 años.

El curso Geometría Plana tiene como propósito que los estudiantes aprendan a demostrar deductivamente. Se emplea una estrategia metodológica que se caracteriza porque, a raíz de problemas abiertos de investigación propuestos por el profesor, los estudiantes (en grupos de dos o tres) se involucran en la resolución (con el apoyo de un EGD) que les exige formular conjeturas y validarlas con elementos del sistema teórico ${ }^{4}$ que se va conformando.

Cada grupo de estudiantes debe presentar sus producciones de manera escrita, incluyendo: i) una narración de cómo se procedió para establecer la solución del problema (e.g., qué se construyó para obtener la configuración que se realizó en el EGD, qué

\footnotetext{
${ }^{4}$ El sistema teórico se corresponde con la propuesta de Birkhoff (1932) en el que se introducen los hechos geométricos encarnados en la regla y el transportador, lo cual hace que la medida subyace al concepto de congruencia. Por ello, los EGD son un modelo pertinente que provee elementos para convencerse empíricamente de la plausibilidad teórica del hecho geométrico que se evidencia.
} 
exploración empírica se hizo, qué tipo de invariante se determinó); y ii) la conjetura (solución del problema) enunciada como proposición condicional que reporta la relación entre lo construido y lo descubierto. A veces se exige consignar un intento de demostración de la conjetura.

El profesor da espacio para que cada grupo presente ante la clase sus producciones. Luego de una discusión en la que participan estudiantes, orientada por el profesor, se escogen las conjeturas que son correctas desde un punto de vista matemático (y que solucionan el problema), se ajustan si es necesario (i.e., se precisan antecedente y consecuente de la conjetura, se formula explícitamente como condicional etc.), y se procede a realizar colectivamente su demostración.

\subsection{Datos para el análisis}

Los datos para el presente reporte son producciones escritas de los estudiantes en las que reportan la solución de tres problemas específicos tomados de Samper y Molina (2013), cuyos enunciados se presentarán más adelante. Estos problemas se escogieron porque son representativos de la tipología que se describirá y porque tenían como propósito involucrar el objeto mediatriz de un segmento ${ }^{5}$ como elemento clave para establecer una solución más completa.

Se contó, además, con otras dos fuentes: i) notas de clase elaboradas después de cada clase por grupos de dos o tres estudiantes, tarea asignada desde el principio del semestre. En ellas, los estudiantes debían reconstruir los principales aspectos tratados en la clase (precisando, por ejemplo, las producciones que los grupos de estudiantes presentan, y los comentarios - del profesor u otros estudiantes - que surgen al respecto de estos) y enviarlas, vía correo electrónico, al profesor, quien luego de revisarlas y corregirlas, las ubicaba en una carpeta virtual en la web a la cual tienen acceso todos los estudiantes del curso; ii) reconstrucción narrativa - método que en inglés se conoce como retrospective recall (SHERIN; RUSS; COLESTOCK, 2011) - hecha por el profesor luego de cada sesión de clase, que permitió complementar la información consignada en las notas de clase.

\footnotetext{
${ }^{5}$ Las caracterizaciones geométricas de dicho objeto son: 1) el lugar geométrico de puntos que equidistan de los extremos del segmento y 2) la recta perpendicular al segmento que contiene su punto medio. Cualquiera de ellas puede ser la definición de mediatriz; la otra sería un teorema. Para este caso, la opción 1 es la definición escogida.
} 


\subsection{Análisis de los datos}

El análisis de los datos consta de dos partes. Un análisis preliminar (de índole teórico), donde se describe, brevemente, cómo debería proceder un estudiante para establecer su solución, según el tipo del problema y se sugiere, a priori, el tipo de argumento que se debe favorecer. Otro retrospectivo (de índole empírico) contrastado con el teórico, donde se identifican tanto las estrategias más comunes de solución de los problemas como sus respectivas conjeturas-solución, evidenciadas en las producciones de los estudiantes. De ambos análisis, obtuvimos insumos para identificar el papel del EGD en el proceso de solución y los tipos de argumentos (inductivos, abductivos y deductivos) que necesariamente se provocan cuando los estudiantes resuelven un tipo particular de problemas. Para el primer caso, empleamos como herramienta analítica los tipos de arrastre (ARZARELLO et al., 2002); para el segundo, el Modelo de Toulmin para un argumento (BOERO et al., 2010).

Vale indicar que, para cada uno de los problemas escogidos, la identificación de los argumentos asociados y los tipos de arrastre que emergieron se hizo a partir de los siguientes datos específicos: para dar cuenta de los tipos de arrastres, principalmente se tuvo en cuenta el reporte escrito de la narración de los estudiantes respecto a cómo ellos procedieron para establecer la solución del problema; esto es, qué construyeron para obtener la configuración que realizaron en el EGD y qué exploración empírica llevaron a cabo para determinar algún invariante.

La reconstrucción hecha por los profesores en relación con las formas de exploración ayudó a complementar la información al respecto. De otro lado, una lectura de estos mismos elementos de los reportes escritos de los estudiantes junto con lo que se evidencia en los documentos notas de clase (que registran la interacción de lo sucedido en cada clase), fueron los datos que nos llevaron a identificar los tipos de argumentos que emergieron de la actividad de los estudiantes.

Lo que presentamos son casos prototípicos construidos a partir de los datos analizados. Para ganar validez en los análisis, en primera instancia estos fueron realizados por cada uno de los autores del artículo, separadamente. Luego, tales análisis se compararon hasta establecer un consenso que fue puesto a consideración de otros académicos conocedores del tema ${ }^{6}$.

\footnotetext{
${ }^{6}$ Ver la sección Agradecimientos.
} 


\section{Relación entre tipos de problemas y tipos de argumentos}

Con el propósito de favorecer la formulación de argumentos inductivos y abductivos, principalmente durante el proceso de conjeturación, el grupo $\mathcal{A} \bullet \mathcal{G}$ ha diseñado diversos tipos de problemas cuya implementación ha sido fuente para sus investigaciones (SAMPER et al., 2010; SAMPER et al., 2014). Con este artículo, concretamente se busca establecer la correspondencia entre tipo de problema y tipo de argumento favorecido.

En los tipos de problemas diseñados (SAMPER et al., 2013) se exige formular la solución como una conjetura expresada como condicional. De acuerdo con la estructura del enunciado del problema, es decir, de la información que aporta el problema y la pregunta que se propone, el resolutor debe encontrar la relación entre conjuntos de propiedades para establecer el antecedente o el consecuente o la proposición condicional. Por esta razón, los problemas se clasifican según el foco de la búsqueda: consecuente, antecedente o determinación de dependencia.

A continuación, se exponen los tipos de problemas, ejemplos de enunciados, una descripción breve sobre lo que deberían hacer los estudiantes para establecer su solución y una descripción detallada de los procesos (o estrategias) de solución representativas que surgieron en las clases de geometría citadas, explicitando los tipos de arrastre que pueden emplearse. En el marco de tal descripción, se presentan los tipos de argumentos que se favorecieron, con lo cual se precisa la correspondencia entre tipo de problema y tipo de argumento que necesariamente debe surgir durante el proceso de solución.

\subsection{Problemas de búsqueda del consecuente}

En este tipo de problemas están dadas condiciones suficientes y se deben hallar las consecuencias necesarias de estas; por ello, se denominan problemas de búsqueda del consecuente. En estos, la representación gráfica de la situación descrita en el enunciado exige la construcción de figuras que cumplan las condiciones dadas; la búsqueda de invariantes (que conformará el consecuente) se hace mediante la exploración de las figuras construidas.

Problema 1: Dados tres puntos no colineales A, B y C. Sea m la mediatriz del $\overline{A B}$ y $n$ la mediatriz del $\overline{B C}$. Sea $T$ el punto de intersección de tales mediatrices. ¿Qué característica geométrica tiene el punto $\mathrm{T}$ al mover el punto B? Formule una conjetura y demuéstrela.

Para solucionar el problema, la representación construida en el EGD debe cumplir las condiciones dadas (en cursiva) en el enunciado (Figura 4). Hecha tal representación, el 
estudiante debe iniciar un proceso de exploración de varios casos (varias posiciones para el punto $B$ ) a través de un arrastre libre para determinar propiedades invariantes del punto $T$. Así, a priori, concebimos que debe surgir un argumento inductivo en el proceso de solución.

Las producciones de los estudiantes mostraron que fue difícil determinar a simple vista las propiedades invariantes mediante el arrastre libre del punto $B$. Propusieron dos estrategias (representativas) para solventar esto: una consistente en marcar el punto $T_{i}$ para posiciones particulares del punto $B$ (denotadas por $B_{i}$ ); en este caso, marcar tres puntos fue suficiente para que los estudiantes visualizaran una invariante: los puntos $T_{i}$ son colineales (Figura 5). La otra estrategia consistió en activar la traza o rastro del punto $T$ y arrastrar libremente al punto $B$; ahí se dieron cuenta que aparece dibujado un segmento de recta (Figura 6).

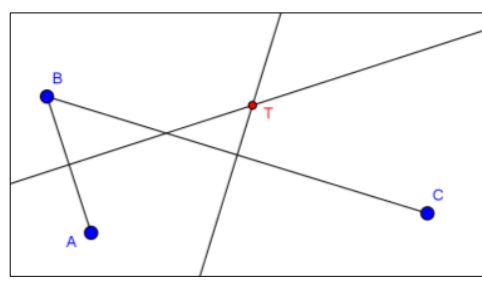

Figura 4 - Representación antecedente Problema 1 Fuente: elaborada por los autores

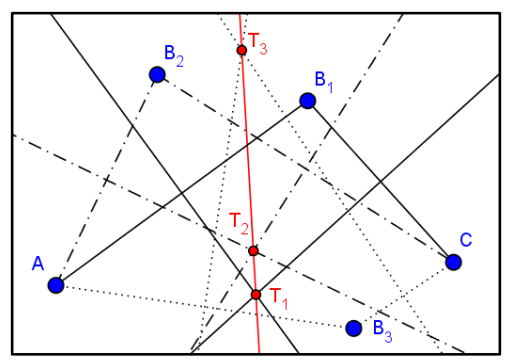

Figura 5 - Primera estrategia de solución Problema 1 Fuente: elaborada por los autores

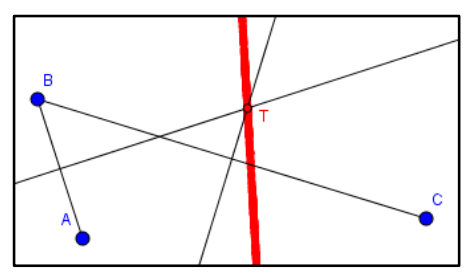

Figura 6 - Segunda estrategia de solución Problema 1 Fuente: elaborada por los autores

La primera estrategia imita una exploración que se puede hacer con papel, lápiz, regla y compás, mientras que la segunda es posible solo en un EGD. Por supuesto, esta última es mucho más significativa por cuanto, en tiempo real, se representan varias posiciones de $B_{i}$ y no solo tres como sucede en el primer caso. Así, con la primera estrategia es posible que solo se infiera la equidistancia de $\operatorname{los}$ puntos $T_{i}$ a los puntos $A$ y $C$, mientras que la segunda estrategia aporta mayor evidencia empírica para inferir que $T_{i}$ es un punto de una recta especial. Estas estrategias permiten establecer una conjetura como resultado de un argumento inductivo, la cual podría estar o no expresada en términos dinámicos. En consecuencia, las conjeturas que se establecen coinciden con la garantía o regla que se induce. Para el caso que estamos reportando, las conjeturas más recurrentes en los reportes de los estudiantes fueron:

Conjetura 1 Dados tres puntos no colineales $A, B$ y $C$. Sea $m$ la mediatriz del $\overline{A B}, n$ la mediatriz del $\overline{B C}$ y $T$ el punto de intersección entre $m$ y $n$. Si $B$ se mueve, entonces $T$ pertenece a la mediatriz del $\overline{A C}$.

Conjetura $2 \mathrm{Si} A, B$ y $C$ son tres puntos no colineales, $m$ es la mediatriz del $\overline{A B}$, $n$ es la mediatriz del $\overline{B C}$ y $T$ el punto de intersección entre $m$ y $n$, entonces $T$ pertenece a la mediatriz $\operatorname{del} \overline{A C}$.

El argumento inductivo, que tuvo lugar en los procesos de solución reportados por los estudiantes, se representa con el Modelo de Toulmin mediante el Diagrama (Figura 7). 


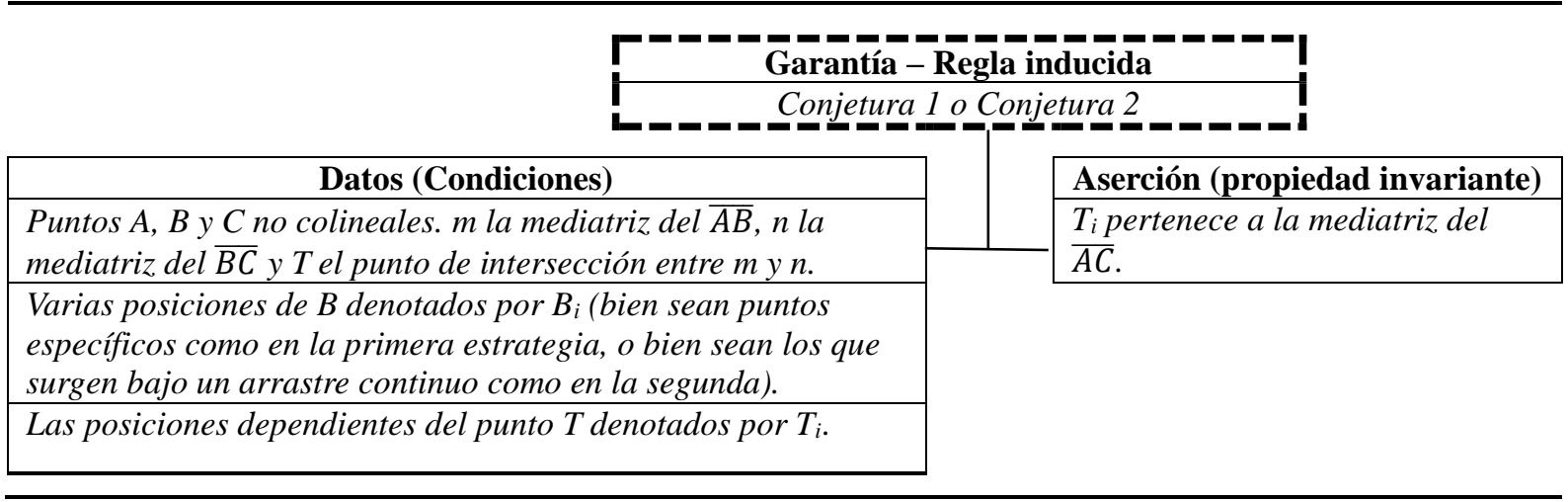

Figura 7 - Diagrama que esboza el argumento inductivo provocado por Problema 1

Fuente: elaborada por los autores

Este ejemplo nos permite concluir que el tipo de problemas búsqueda del consecuente favorece la producción de un argumento inductivo: el resolutor toma varios casos que cumplen ciertas condiciones dadas (fuente cursiva en el enunciado del problema), observa (empíricamente) una propiedad invariante ( $T$ pertenece a la mediatriz del $\overline{A C}$ ) que depende de tales condiciones, e induce una regla general (conjetura). El EGD favorece de mejor manera la inducción pues vía el arrastre libre es posible visualizar la propiedad invariante para infinitos casos.

\subsection{Problemas de búsqueda del antecedente}

En este tipo de problema se deben hallar las condiciones suficientes para las cuales las propiedades mencionadas en el enunciado son la consecuencia necesaria; por ello se denominan problemas de búsqueda del antecedente. Resolverlos exige no sólo representar gráficamente los objetos mencionados en el enunciado, sino también realizar construcciones auxiliares que provean las condiciones geométricas suficientes para reconocer, mediante la exploración, las propiedades de un objeto existente o determinar las que aseguren la existencia de un objeto. Estas deben ser reportadas en el antecedente de la conjetura pues el consecuente está dado.

Problema 2: Sean $\overline{A B}$ y $\overline{C D}$ dos segmentos congruentes. ¿Es posible determinar un punto $\mathrm{E}$ de forma tal que $\triangle \mathrm{ABE}$ y $\triangle \mathrm{CDE}$ sean congruentes? Provea una conjetura y demuéstrela.

El resolutor debe hacer una representación en el EGD que satisfaga las condiciones (en cursiva) dadas en el enunciado, y buscar condiciones del punto $E$ para obtener como consecuencia necesaria la congruencia del $\triangle A B E$ y $\triangle C D E$. Precisamente, es necesario establecer parte del antecedente de la conjetura que sería la solución del problema. Es decir, 
debe completar la siguiente proposición abierta para que sea verdadera:

Si $\overline{A B}$ y $\overline{C D}$ son dos segmentos congruentes y $E$ es un punto tal que , entonces $\triangle A B E$ y $\triangle C D E$ son congruentes.

A priori, concebimos que este tipo de problema favorece la producción de un argumento abductivo durante el proceso de conjeturación. Es probable, también, que los estudiantes usen un arrastre libre de los extremos de los segmentos o de un punto $E$ buscando una solución, un arrastre guiado (e.g., poner los segmentos dados en una posición específica), un arrastre de lugar ficticio (e.g., mover el punto $E$ intentando mantener los triángulos en cuestión congruentes sin darse cuenta de la trayectoria seguida por tal punto) o un arrastre mantenido (e.g., hacer lo mismo, pero percatándose de la trayectoria seguida por el punto E).

Los registros que analizamos muestran que los estudiantes procedieron de distintas formas válidas. Unos adjudicaron una relación particular a los $\overline{A B}$ y $\overline{C D}$ (e.g., que fueran paralelos entre sí, que los segmentos se bisecaran, que fueran perpendiculares etc.), y evocaron los criterios de congruencia de triángulos. Por ejemplo, unos estudiantes asumieron que $\overline{A B} \| \overline{C D}$ (siguiendo un arrastre guiado) y tomaron a $E$ como la intersección de las diagonales del $\square A B C D$ para dar una solución al problema (Figura 8).

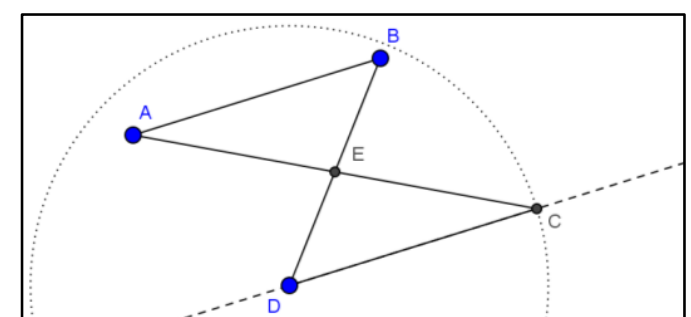

Figura 8 - Primera estrategia de solución problema 2 Fuente: elaborada por los autores

Pero ¿por qué asumieron que $\overline{A B}$ y $\overline{C D}$ son paralelos entre sí? Según su relato, evocaron dos hechos geométricos conocidos por ellos que permitirían establecer una nueva meta (submeta 1): la congruencia de los lados correspondientes de los triángulos involucrados en la situación $(\overline{A E} \cong \overline{E C}, \overline{B E} \cong \overline{E D}$ ) para, así, obtener (posiblemente usando el criterio Lado-Lado-Lado) la congruencia de los triángulos, meta del problema. Evocaron el hecho: si un cuadrilátero es paralelogramo entonces sus diagonales se bisecan. Esto les conducía a tener como un dato nuevo: un paralelogramo para el cual dos de sus lados eran los segmentos dados. Reconocemos en esto la producción de un argumento abductivo (Diagrama de la Figura 9). Para obtener el dato nuevo, evocaron otro hecho: si los lados opuestos de un cuadrilátero son congruentes y paralelos, entonces es un paralelogramo. Esto para determinar datos nuevos sobre los segmentos dados que llevaran a configurar un paralelogramo (submeta 2). De nuevo reconocemos un argumento abductivo (Diagrama de la 
Figura 10).

\begin{tabular}{|c|c|}
\hline \multicolumn{2}{|c|}{ Garantía - Regla evocada } \\
\hline Si $\triangle A B C D$ es un paralelogramo, entonces sus diagonales se bisecan. \\
\hline $\begin{array}{c}\square A B C D \text { es un paralelogramo, (y E es la } \\
\text { intersección de sus diagonales) }\end{array}$ \\
\hline
\end{tabular}

Figura 9 - Diagrama que esboza el primer argumento abductivo provocado por Problema 2, primera estrategia Fuente: elaborada por los autores

\begin{tabular}{|c|c|}
\hline \multicolumn{2}{|c|}{ Garantía - Regla evocada } \\
\hline \multicolumn{2}{|c|}{ Si los lados opuestos de un cuadrilátero son congruentes y paralelos, entonces es un paralelogramo. } \\
\hline \\
\hline$\overline{A B}$ y $\overline{C D}$ son congruentes y paralelos y E es \\
su intersección
\end{tabular}

Figura 10 - Diagrama que esboza el segundo argumento abductivo provocado por Problema 2, primera estrategia

Fuente: elaborada por los autores

Finalmente, los estudiantes formularon la siguiente conjetura como solución al problema 2: $\overline{A B}$ y $\overline{C D}$ son congruentes y paralelos y $E$ es la intersección de $\overline{A C}$ y $\overline{B D}$, entonces $\triangle A B E$ y $\triangle C D E$ son congruentes. Además, si los estudiantes realmente usaron el criterio Lado-Lado-Lado (en su relato no lo explicitaron) para asegurar la congruencia de los triángulos (meta del problema), se habría dado un argumento deductivo (Diagrama de la Figura $\left.11^{7}\right)$.

\begin{tabular}{|c|c|}
\hline \multicolumn{2}{|c|}{ Garantía - Regla evocada (conocida) } \\
\hline \multicolumn{2}{|c|}{ Criterio Lado-Lado-Lado de congruencia de triángulos } \\
\hline Datos & Aserción - meta \\
\hline$\overline{A B} \cong \overline{C D}, \overline{A E} \cong \overline{E C}, \overline{B E} \cong \overline{E D}$ & $\triangle A B E$ y $\triangle C D E$ son congruentes \\
\hline
\end{tabular}

Figura 11 - Diagrama que esboza el posible argumento deductivo provocado por Problema 2, primera estrategia

Fuente: elaborada por los autores

La segunda estrategia que evidenciamos consistió en buscar el punto $E$ con el arrastre de lugar ficticio, es decir, sin caracterizarlo geométricamente. Los estudiantes realizaron exploraciones buscando la congruencia de parejas de segmentos $o$ de ángulos correspondientes. Varios grupos de estudiantes, por ejemplo, reportaron que querían

\footnotetext{
${ }^{7}$ No aceptar como solución final relaciones particulares para tales segmentos, implica un mayor nivel de complejidad del problema. Una complejidad aún mayor implica considerar la situación en la cual $\overline{A B}$ y $\overline{C D}$ están en planos diferentes (i.e., las rectas que los contienen son alabeadas).
} 
establecer que el $\overline{A E}$ fuera congruente al $\overline{C E}$ o el $\overline{B E}$ al $\overline{D E}$. Algunos de ellos reconocieron que, para que ello sucediera, el punto $E$ debería estar en la mediatriz del $\overline{A C}$, objeto geométrico que ya conocían.

Otros usaron una estrategia basada en el arrastre mantenido, tomando un punto $E$ cualquiera, activando su traza, y moviéndolo con el objetivo de mantener la propiedad que deseaban (i.e., congruencia de $\overline{A E}$ con $\overline{C E}$ ); dicha traza les permitió reconocer que el punto $E$ debería estar en la mediatriz del $\overline{A C}$ (Figura 5). Luego, como lo afirmaron ambos grupos de estudiantes, producto de un análisis similar, determinaron que $E$ también debería estar en la mediatriz del $\overline{B D}$. Así, establecieron que la congruencia de los triángulos se lograba cuando $E$ era la intersección de las mediatrices (Figura 12). El enunciado formulado como conjeturasolución fue similar a: Si $\overline{A B}$ y $\overline{C D}$ son congruentes y $E$ es el punto de intersección de las mediatrices de $\overline{A C}$ y $\overline{B D}$, entonces $\triangle A B E$ y $\triangle C D E$ son congruentes.

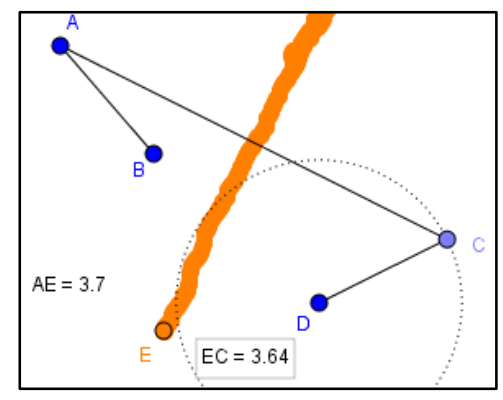

Figura 12 - Segunda estrategia de solución problema 2 con arrastre mantenido

Fuente: elaborada por los autores

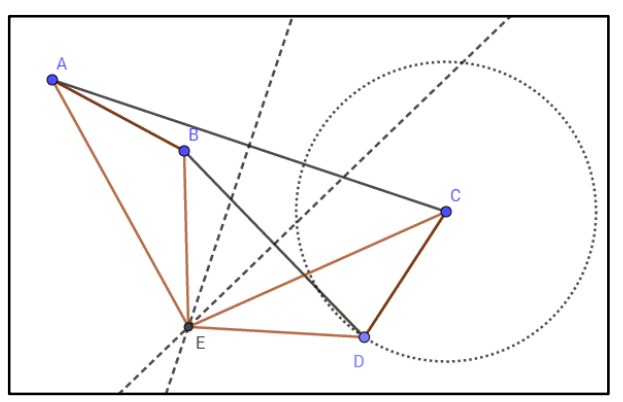

Figura 13 - Representación solución problema 2 Fuente: elaborada por los autores

Las acciones realizadas en la segunda estrategia de solución seguramente obedecieron a un argumento abductivo (Diagrama de la Figura 14).

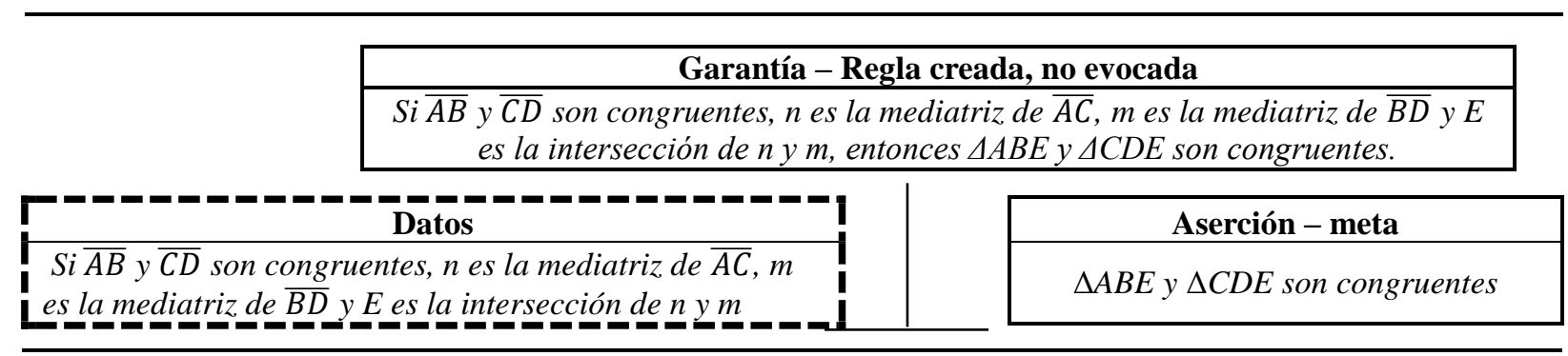

Figura 14 - Diagrama que esboza el argumento abductivo provocado por Problema 2, segunda estrategia Fuente: elaborada por los autores

Aunque los procesos llevados a cabo en ambas estrategias favorecen la producción de argumentos abductivos, existe una diferencia fundamental entre ellos. El proceso de la segunda estrategia tiene un carácter creativo, pues el resolutor descubre, producto de su exploración empírica basada en el arrastre mantenido, posibles datos que llevarían a la congruencia de los triángulos. En contraste, el proceso de la primera estrategia se apoya en 
una evocación teórica de un hecho geométrico conocido por los estudiantes cuyo consecuente se corresponde con la congruencia de los triángulos. Así, unos argumentos que surgen para resolver el problema están fundados en experimentos empíricos, otros en evocaciones de hechos teóricos conocidos.

\subsection{Problemas de determinación de dependencia}

En un problema de determinación de dependencia - análogo a una de las tipologías citadas por Yevdokimov (2004) - el enunciado provee un conjunto referencial de figuras geométricas y unas propiedades, y solicita establecer dependencias entre "tipos de figuras del conjunto referencial" y las "propiedades dadas". Existe la libertad de decidir qué se toma como antecedente (o consecuente) de la conjetura: el conjunto referencial o las propiedades.

Problema 3: Estudie la relación entre el tipo de cuadrilátero y propiedad las mediatrices de dos de sus lados coinciden ${ }^{8}$. Provea una conjetura y demuéstrela.

En un problema como este, el resolutor podría realizar por lo menos dos estrategias para abordarlo: i) asumir como antecedente tipos de cuadriláteros o ii) asumir como antecedente la propiedad las mediatrices de dos de sus lados coinciden. En cualquiera de los dos casos, se debería construir, blandamente (mediante arrastre guiado) o robustamente ${ }^{9}$, en el EGD las propiedades del antecedente escogido. Para el primer caso, se esperaría que el resolutor estudie varios casos del tipo de cuadrilátero escogido, a través del arrastre libre, y observe lo que sucede con sus mediatrices buscando invariantes (en especial, la propiedad del enunciado); lo deseable sería que hiciere el mismo proceso de exploración con cada uno de los tipos de cuadriláteros (cuadrado, rectángulo, paralelogramo, rombo, cometa, trapecios isósceles o no, cuadrilátero cíclico) y de todos ellos generalizase para formular una conjetura representativa de todos los casos. Para la segunda estrategia, lo deseable sería que el resolutor observe lo que sucede con el cuadrilátero determinado por los extremos de los segmentos, cuando las mediatrices coinciden, para formular una conjetura general.

Evidenciamos las estrategias anteriormente descritas en la producción de los estudiantes; la primera tiene mayor frecuencia que la segunda; además, la tendencia para hacer la exploración es usar configuraciones blandas más que construcciones robustas. Así,

\footnotetext{
${ }^{8}$ Que dos rectas coincidan significa que comparten todos sus puntos.

${ }^{9}$ Por supuesto, si hacen construcciones robustas de los cuadriláteros, ello indica un mayor nivel de complejidad en el desarrollo de la heurística, puesto que implica tener en cuenta propiedades que garanticen que cada representación sea el cuadrilátero escogido bajo el arrastre.
} 
por ejemplo, para la primera estrategia varios grupos de estudiantes realizaron configuraciones blandas de cuadrados, rectángulos, trapecios, paralelogramos y cometas ninguno aludió a un cuadrilátero cíclico - (Figura 15); las conjeturas-solución producidas fueron como las siguientes:

Conjetura 1 Si un cuadrilátero es un rectángulo, entonces las mediatrices de lados opuestos coinciden.

Conjetura 2 Si un cuadrilátero es un trapecio isósceles, entonces las mediatrices de sus lados paralelos coinciden.

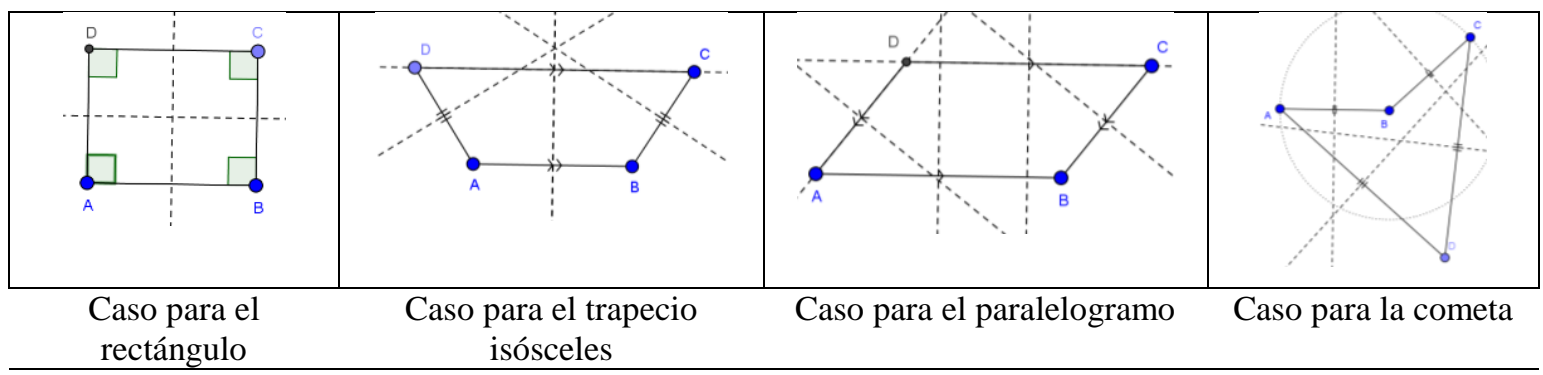

Figura 15 - Primera estrategia problema 3. Algunos casos para tipos de cuadrilátero

Fuente: elaborada por los autores

Quienes usaron la segunda estrategia hicieron como configuración un cuadrilátero con sus mediatrices; arrastraron uno de sus vértices hasta que coincidieran las mediatrices de lados opuestos - i.e., hicieron un arrastre guiado - (Figura 16). Formularon conjeturas-solución como: Si en un cuadrilátero las mediatrices de dos de sus lados coinciden, entonces es un rectángulo o un trapecio isósceles.

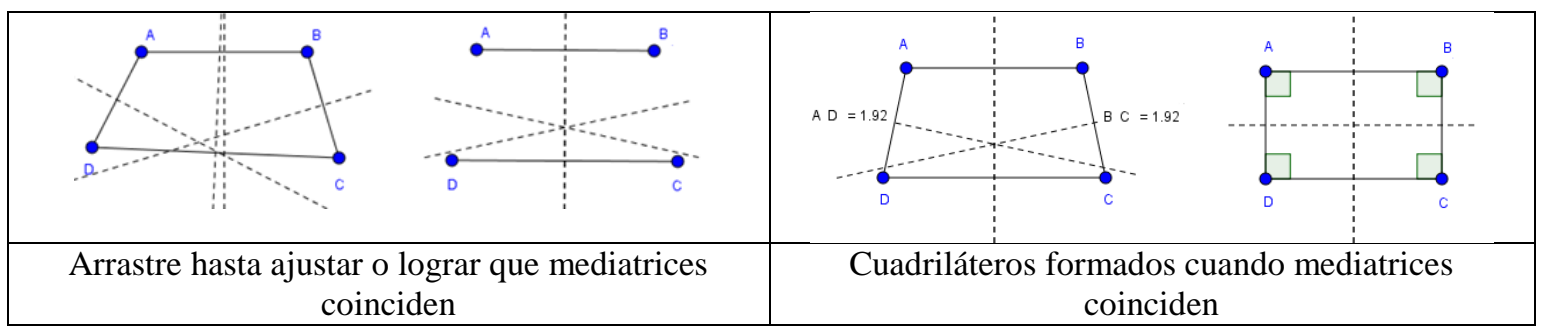

Figura 16 - Segunda estrategia problema 3. Caso construcción blanda Fuente: elaborada por los autores

A partir de estos ejemplos, se evidencia que el argumento que se favorece con la estrategia 1 es abductivo: se conoce el resultado y se quiere determinar qué propiedades causan ese resultado. En cambio, con la segunda estrategia el argumento es de carácter inductivo, pues sucede algo similar a lo descrito para los problemas de búsqueda de consecuente. En consecuencia, tenemos insumos para concluir que el tipo de problemas de determinación de dependencia favorece la formulación de argumentos inductivos y abductivos. No obstante, un análisis más fino da la posibilidad de que se favorezcan argumentos abductivos y deductivos.

Veamos el caso de un grupo de estudiantes que, en el marco de la primera estrategia, 
hicieron una construcción robusta para tipos de cuadriláteros. En su relato, reportaron que pensaron en las propiedades que debían construir para obtener un tipo especial de cuadrilátero (e.g., cometa) y determinaron sí se cumplía la propiedad solicitada; vemos en este proceder un argumento abductivo que esquematizamos en el diagrama expreso por la Figura 17. Su conjetura solución fue: Si $\overleftrightarrow{A C}$ es mediatriz de $\overline{B D}$ entonces $\square A B C D$ es una cometa. Para la segunda estrategia, no reportaron exploraciones con una construcción robusta; si lo hubieran hecho, habría surgido un argumento abductivo (un ejemplo, se esquematiza en el diagrama expreso en la Figura 18).

\begin{tabular}{|c|c|c|c|}
\hline \multirow{2}{*}{\multicolumn{2}{|c|}{$\begin{array}{l}\text { Garantía - Regla conocida o no } \\
\text { Si en un cuadrilátero, la recta que } \\
\text { contiene una de sus diagonales es } \\
\text { mediatriz de la otra, entonces el } \\
\text { cuadrilátero es cometa }\end{array}$}} & \multicolumn{2}{|c|}{ Garantía - Regla conocida o no } \\
\hline & & \multicolumn{2}{|c|}{$\begin{array}{l}\text { Si } A \text { y } D \text { están del mismo lado de la recta } m, \text { y } B \text { y } \\
C \text { son las reflexiones axiales de } A \text { y } D \text { respecto a } \\
\text { m, entonces } m \text { es la mediatriz de } \overline{A B} \text { y } \overline{C D} \text {. }\end{array}$} \\
\hline Datos posibles & Aserción - meta & \multirow{2}{*}{$\begin{array}{l}\text { Datos posibles } \\
\text { A y } D \text { están del mismo } \\
\text { lado de } m \text {; } B \text { y } C \text { son } \\
\text { las reflexiones axiales } \\
\text { de A y } D \text { respecto a } m\end{array}$} & Aserción - meta \\
\hline $\begin{array}{c}\text { Si } \overleftrightarrow{A C} \text { es } \\
\text { mediatriz de } \\
\overrightarrow{B D}\end{array}$ & $\begin{array}{l}\square A B C D \text { es una } \\
\text { cometa }\end{array}$ & & $\begin{array}{l}\text { mes la mediatriz } \\
\quad \text { de } \overline{A B} \text { y } \overline{C D}\end{array}$ \\
\hline \multicolumn{2}{|c|}{$\begin{array}{c}\text { Figura } 17 \text { - Argumento abductivo provocado } \\
\text { por Problema 3, primera estrategia }\end{array}$} & \multicolumn{2}{|c|}{$\begin{array}{c}\text { Figura } 18 \text { - Argumento abductivo provocado } \\
\text { por Problema 3, segunda estrategia }\end{array}$} \\
\hline
\end{tabular}

Fuente: elaborada por los autores

Por otro lado, los argumentos deductivos tuvieron lugar cuando, en uno de los cursos, se propuso como tarea producir una sola conjetura que generalizara los resultados reportados en las conjeturas enunciadas resultado de la primera estrategia; ello implicó analizar los antecedentes de estas (pues el consecuente en ambas es el mismo). Los estudiantes establecieron las propiedades comunes del rectángulo y del trapecio isósceles: dos de sus lados son paralelos y los ángulos adyacentes de la base son congruentes. De este suceso, entrevemos la producción de dos argumentos deductivos (diagramas expresos en las Figuras 19 y 20).

\begin{tabular}{|c|c|c|c|}
\hline \multirow{2}{*}{\multicolumn{2}{|c|}{\begin{tabular}{|c|} 
Garantía - Regla conocida \\
Si un cuadrilátero es un rectángulo, \\
entonces, $i$ ) sus lados opuestos son \\
paralelos, ii) sus ángulos son \\
congruentes. \\
\end{tabular}}} & \multicolumn{2}{|c|}{ Garantía - Regla conocida } \\
\hline & & \multicolumn{2}{|c|}{$\begin{array}{c}\text { Si un cuadrilátero es un trapecio isósceles, } \\
\text { entonces i) sus lados opuestos no paralelos son } \\
\text { congruentes, ii) los ángulos de la base son } \\
\text { congruentes. }\end{array}$} \\
\hline Datos & Aserción - meta & Datos & Aserción - meta \\
\hline $\begin{array}{l}\square A B C D \text { es } \\
\text { un } \\
\text { rectángulo }\end{array}$ & $\begin{array}{c}\overline{A B}\|\overline{C D}, \overline{B C}\| \overline{A D} \\
\mathrm{y} \angle C \cong \angle D \cong \angle B \cong \\
\angle A\end{array}$ & $\begin{array}{l}\square A B C D \text { es un } \\
\text { trapecio } \\
\text { isósceles }\end{array}$ & $\begin{array}{c}\overline{A B} \| \overline{C D}, \overline{B C} \cong \overline{A D} \\
\mathrm{y} \angle C \cong \\
\angle D, \angle A \cong\end{array}$ \\
\hline \multicolumn{2}{|c|}{$\begin{array}{c}\text { Figura } 19 \text { - Argumento deductivo provocado } \\
\text { por Problema 3, primera estrategia }\end{array}$} & \multicolumn{2}{|c|}{$\begin{array}{c}\text { Figura } 20 \text { - Argumento deductivo provocado } \\
\text { por Problema 3, segunda estrategia }\end{array}$} \\
\hline
\end{tabular}


De las aserciones de tales argumentos deductivos (ver lo sombreado), fue generalizado el antecedente de la nueva conjetura-solución del problema 3. La reformulación propuesta fue: Si es un cuadrilátero dos de sus lados son paralelos y los ángulos adyacentes de la base son congruentes, entonces las mediatrices de sus lados paralelos coinciden.

\section{Discusión y reflexiones finales}

Se han descrito y analizado resultados de una experiencia implementada en un programa de formación de profesores de matemática en la cual, siguiendo las ideas de Stylianides y Stylianides (2006), y Stylianides y Ball (2008), se involucró una tipología de tareas (problemas abiertos de conjeturación) que fomentan actividad argumentativa.

Se ilustró, con ejemplos específicos de problemas y de estrategias (o heurísticas) empleadas en un EGD para resolver los problemas, cómo el tipo de problema provoca la generación de ciertos tipos argumentos durante el proceso de resolución (que lleva a la formulación de una conjetura). Se identificaron y describieron los tipos de argumentos asociados siguiendo el Modelo de Toulmin (BOERO et al., 2010). Con lo anterior, se tiene un escenario que da pie para proponer una correspondencia entre el tipo de argumento y el tipo de problema (Cuadro 1), aunque ello no implica que un tipo de problema genere exclusivamente algún tipo de argumento.

\begin{tabular}{|c|c|c|c|c|}
\hline $\begin{array}{c}\text { Tipo de } \\
\text { Problema }\end{array}$ & $\begin{array}{l}\text { Estructura de } \\
\text { enunciado }\end{array}$ & Proceso de resolución & $\begin{array}{c}\text { Estructura de } \\
\text { conjetura }\end{array}$ & $\begin{array}{c}\text { Tipo de } \\
\text { Argumento }\end{array}$ \\
\hline $\begin{array}{l}\text { Búsqueda de } \\
\text { consecuente }\end{array}$ & $\begin{array}{l}\text { Exposición de datos } \\
\text { dados } \rightarrow \text { Pregunta } \\
\text { sobre efecto de ellos }\end{array}$ & $\begin{array}{l}\text { Representación de datos dados } \\
\rightarrow \text { exploración de casos para } \\
\text { determinar consecuencia de } \\
\text { datos dados }\end{array}$ & $\begin{array}{l}\text { Si datos dados, } \\
\text { entonces } \\
\text { generalización de } \\
\text { propiedad } \\
\text { invariante. }\end{array}$ & Inductivo \\
\hline $\begin{array}{l}\text { Búsqueda de } \\
\text { antecedente }\end{array}$ & $\begin{array}{l}\text { Consignación de } \\
\text { propiedad esperada } \\
\rightarrow \text { Pregunta sobre } \\
\text { qué propiedades } \\
\text { pueden causarla }\end{array}$ & $\begin{array}{l}\text { Exploración de propiedades } \\
\text { que pueden causar lo } \\
\text { esperado } \rightarrow \text { verificación de lo } \\
\text { esperado }\end{array}$ & $\begin{array}{l}\text { Si propiedades } \\
\text { encontradas, } \\
\text { entonces } \\
\text { propiedad dada. }\end{array}$ & Abductivo \\
\hline \multirow{2}{*}{$\begin{array}{l}\text { Determinación } \\
\text { de } \\
\text { dependencia }\end{array}$} & \multirow{2}{*}{$\begin{array}{l}\text { Se proveen conjunto } \\
\text { referencial de figuras } \\
\text { geométricas y ciertas } \\
\text { propiedades. Solicita } \\
\text { establecer } \\
\text { dependencias entre } \\
\text { p: tipos de figuras } \\
\text { del conjunto } \\
\text { referencial y } \\
\text { q: propiedades } \\
\text { dadas. }\end{array}$} & $\begin{array}{l}\text { Si se asume } p \text { o } q \text { como dato } \\
\text { dado, y se busca una } \\
\text { consecuencia asociada a } q \text { o } \\
p \text {, respectivamente, el proceso } \\
\text { es igual a Búsqueda de } \\
\text { consecuente. }\end{array}$ & $\begin{array}{l}\text { Si datos dados, } \\
\text { entonces } \\
\text { generalización de } \\
\text { propiedad } \\
\text { invariante. }\end{array}$ & Inductivo \\
\hline & & $\begin{array}{l}\text { Si se asume } p \text { o } q \text { como } \\
\text { propiedad esperada dada, y se } \\
\text { pregunta si } q \text { o } p \\
\text { respectivamente, puede } \\
\text { causarla, el proceso es igual a } \\
\text { Búsqueda de antecedente. }\end{array}$ & $\begin{array}{l}\text { Si "propiedades } \\
\text { encontradas", } \\
\text { entonces } \\
\text { "propiedad } \\
\text { dada". }\end{array}$ & Abductivo \\
\hline
\end{tabular}

Cuadro 1 - Correspondencia entre tipo de problema con tipo de argumento

Fuente: elaboradas por los autores 
El Cuadro 1 pone en evidencia cómo la estructura del enunciado provoca el proceso de resolución en tanto indica claramente lo que se debe considerar como dado y lo que se debe descubrir; en tal sentido, infunde qué parte de la conjetura-solución (antecedente o consecuente) está dada y cuál debe establecerse. En otras palabras, y empleando los términos del modelo de Toulmin en un problema de búsqueda de antecedente, los datos están dados y se debe realizar una exploración sobre ellos que dejaría notar una propiedad invariante (aserción); en consecuencia, se podría inducir, empíricamente, una regla general que se convierte en la conjetura-solución o garantía susceptible de ser estudiada para convertirse en teorema. De otro lado, en un problema de búsqueda de antecedente, la aserción está dada y se debe evocar o descubrir una proposición (garantía) que contenga como consecuente dicha aserción; por lo tanto, se podría abducir unos datos que se corresponde con el antecedente de tal proposición y que deben ser legitimados por el contexto de la situación. La proposición garantía se convierte en la conjetura-solución susceptible de ser estudiada para convertirse en teorema (en caso de que no lo sea). Como un problema de determinación de dependencia se convierte en alguno de los otros tipos anteriores según su abordaje, los argumentos inductivos o abductivos emergen de la misma forma descrita.

Dado que los datos analizados se focalizaron en proceso de exploración para formular conjeturas que solucionan problemas, la referencia a los argumentos deductivos fue escasa. Ello va en consonancia con la idea de varios autores (ARZARELLO et al., 2012; BACCAGLINI-FRANK; MARIOTTI, 2010; PEDEMONTE, 2007) según la cual los argumentos informales de índole abductivo o inductivo toman lugar en procesos de exploración como una forma para soportar las conjeturas que se formulan.

Ahora, si bien ciertos tipos de problemas provocaron ciertos tipos de argumentos (ver Cuadro 1), debemos indicar que la metodología empleada en el aula contribuyó, también, a la producción de argumentos de diversa índole. Como se mostró, el uso de EGD posibilitó una mayor riqueza en la exploración de las situaciones; específicamente, los tipos de arrastre libre y guiado estuvieron presentes en todos los tipos de problemas, y fueron, principalmente, el soporte para argumentos inductivos. Se destaca el uso del arrastre mantenido (no tan frecuente como los otros) como soporte para los argumentos abductivos, sugerido por el problema tipo búsqueda de antecedente.

Los resultados del análisis ratifican un cierto éxito de la innovación en el aula implementada por el grupo Aprendizaje y Enseñanza de la Geometría (UPN, Colombia) en un programa de formación de profesores. La terna (problemas abiertos de conjeturación, EGD, interacción en el aula/argumentación) descrita al inicio del artículo, se puede caracterizar 
como un elemento que contribuye a un ambiente de clase en el que el futuro profesor tiene espacio no solo para hacer una actividad matemática cercana a la de un matemático profesional (en términos de explorar una situación, conjeturar, y argumentar), sino para experimentar en propia piel el potencial de dicha terna para llevar a cabo dicha actividad.

De manera más concreta, los profesores en formación se involucraron en el proceso de resolver los problemas porque la metodología propuesta en la innovación lo exige: fue a partir de sus conjeturas y las conversaciones matemáticas asociadas, para aceptar o rechazar las ideas matemáticas expuestas, que se organizó el contenido matemático en las clases de geometría. Formularon argumentos de diferentes tipos durante el proceso de resolución, cuando expusieron públicamente sus conjeturas, verificaron sus resultados e intentaron soportar la veracidad de sus conjeturas.

No cabe duda de que con la tipología de problemas se está atendiendo a la formación matemática de los futuros profesores, puesto que realizan actividad matemática: argumentar, resolver, representar, conjeturar, etc. Pero, como abogan Stylianides y Stylianides (2006) y Stylianides y Ball (2008), para que los profesores en formación ganen conocimiento sobre la argumentación, necesario para favorecer ese proceso en sus clases, se requiere realizar conversaciones matemáticas acerca de las facetas didácticas que puede tener el abordaje de tal tipología de problemas en los programas de formación.

En tal sentido, sería necesario destacar en clase la relación entre tipos de problemas y tipos de argumentos y, en ese marco, desarrollar la habilidad de futuro profesores para identificar y clasificar cada argumento, reconocer el papel de estos en la actividad matemática desplegada - inductivos y abductivos para conjeturar, deductivo para validar -, y analizar la estructura de los enunciados de los problemas. Creemos que así los futuros maestros pueden ganar conocimiento de elementos importantes sobre la actividad demostrativa.

Quisiéramos creer que, con lo que aquí exponemos, damos respuesta parcial a la inquietud que Stylianides y Ball (2008) consignan al final de su artículo: ¿cómo pueden los formadores de profesores escolares ayudar a que ellos desarrollen elementos del conocimiento de la demostración considerados importantes que los empodere para que lleven al aula escolar la argumentación? Asegurarlo claramente requiere realizar un estudio investigativo.

Sin embargo, declaraciones como los que siguen son evidencia de que son los mismos profesores en formación que consideran que la innovación implementada, basada en la terna descrita, es un escenario que les provee herramientas para que en su futura labor profesional involucre, en su enseñanza, la resolución de problemas como medio (ZIMMERMANN, 2016) para desarrollar procesos como la exploración, la conjeturación y la argumentación. 
Andrés: Siempre he tenido un especial afecto por los cursos de geometría por la forma en que se enseñan, incluso he intentado llevar esa metodología a las prácticas [i.e., prácticas docentes exigidas por el programa de licenciatura en la que los estudiantes actúan como profesores en aulas reales de secundaria], ya que para mí ayuda a potenciar no solo el aprendizaje de conceptos, sino que también desarrolla la argumentación.

Sonia: Tengo muy presente la forma en que estaba diseñada cada tarea [problema], en donde cada uno representaba para mí un reto, y me daba mucha curiosidad de saber qué íbamos a encontrar; además, que de esta forma no se condiciona a pensar en una única solución, nos ayuda a ir más allá y descubrir diversas soluciones, diferentes formas de crear un sistema teórico. Destaco esto porque me ha ayudado a pensar en los múltiples problemas que uno podría diseñar para mis futuros estudiantes; en donde las preguntas sean abiertas y lo importante que es tomarse el tiempo de pensar en las preguntas y en desarrollar una buena clase en donde los alumnos se exijan y que se diviertan haciendo matemáticas.

(Entrevista hecha al final del curso, 2014).

\section{Agradecimientos}

Un agradecimiento a la Doctora Adriana Breda y a miembros del grupo de investigación $\mathcal{A} \bullet \mathcal{G}$ (UPN, Colombia) por la lectura de una versión previa de este escrito. De igual forma, a los evaluadores de las primeras versiones del documento por sus contribuciones al mismo.

\section{Referencias}

ARZARELLO, F. et al. A cognitive analysis of dragging practises in Cabri environments. ZDM, Germany, v. 34, n. 3, p. 66-72, 2002.

ARZARELLO, F. et al. Experimental Approaches to Theoretical Thinking: Artefacts and Proofs. In: HANNA, G.; DE VILLIERS, M. Proof and Proving in Mathematics Education. New York: Springer, 2012. p. 97-146.

BACCAGLINI-FRANK, A.; MARIOTTI, M. Generating Conjectures in Dynamic Geometry: The Maintaining Dragging Model. International Journal of Computers for Mathematical Learning, Netherlands, v. 15, p. 225-253, 2010.

BACCAGLINI-FRANK, A.; MARIOTTI, M.; ANTONINI, S. Different Perceptions of Invariants and Generality of Proof in Dynamic Geometry. In: TZEKAKI, M.; SAKONIDIS, H. CONFERENCE OF THE INTERNATIONAL GROUP FOR THE PSYCHOLOGY OF MATHEMATICS EDUCATION, 33., 2009, Thessaloniki. Proceedings... Thessaloniki, Greece: PME, v. 2, 2009. p. 89-96.

BALL, D. L.; BASS, H. Making mathematics reasonable in school. In: KILPATRICK, J.; MARTIN, W. G.; SCHIFTER, D. A research companion to Principles and Standards for School Mathematics. Reston, VA: National Council of Teachers of Mathematics, 2003. p. 27-44.

BALL, D.; LUBIENSKI, S.; MEWBORN, D. Research on teaching mathematics: The unsolved problem of teachers' mathematical knowledge. In: RICHARDSON, V. Handbook of research on teaching. 4. ed. New York, NY: Macmillan, 2001. p. 433-456.

BIRKHOFF, G. A set of postulates for plane geometry, based on scale and protractor. Annals of Mathematics, v. 33, n. 2, p. 329-345, 1932. Disponivel em: 〈http://www.jstor.org/stable /1968336〉. Acesso em: Febrero de 2017. 
BOERO, P. et al. Argumentation and proof: a contribution to theoretical perspectives and their classroom implementation. In: CONFERENCE OF THE INTERNATIONAL GROUP FOR THE PSYCHOLOGY OF MATHEMATICS EDUCATION, 34., 2010, Belo Horizonte. Proceedings... Belo Horizonte, Brazil: PME, 2010. p. 179-204.

CAMARGO, L. et al. Use of dragging as organizer for conjecture validation. In: CONFERENCE OF THE INTERNATIONAL GROUP FOR THE PSYCHOLOGY OF MATHEMATICS

EDUCATION, 33., 2009, Thessaloniki. Proceedings... Thessaloniki, Greece: PME, 2009. p. 257-264.

FELMER, P. et al. La resolución de problemas en la matemática escolar y en la formación inicial docente. Estudios de política educativa, Chile, v. 1, n. 1, p. 66-105, 2015.

LABORDE, C. Dynamic Geometry Environments as a Source of Rich Learning Contexts for the Complex Activity of Proving, Netherlands, v. 44, n. 1, p. 151-161, 2000.

LESH, R.; ZAWOJEWSKI, J. Problem solving and modeling. In: LESTER, F. Second Handbook of Research on Mathematics Teaching and Learning. Charlotte, NC: Information Age Publishing, 2007. p. 763-804.

LIN, F. L. et al. Teachers' Professional Learning of Teaching Proof and Proving. In: HANNA, G.; DE VILLIERS, M. Proof and Proving in Mathematics Education. New York: Springer, 2012. p. 327346.

MARIOTTI, M. Proof and Proving in Mathematics Education. In: GUTIÉRREZ, A.; BOERO, P. Handbook of Research on the Psychology of Mathematics Education. Past, Present and Future. Rotterdam, The Netherlands: Sense Publishers, 2006. p. 173-204.

ONUCHIC, L. R.; ALLEVATO, N. S. G. Pesquisa em Resolução de Problemas: caminhos, avanços e novas perspectivas. Bolema, Rio Claro, Brasil, v. 25, n. 41, p. 73-98, 2011.

PEDEMONTE, B. How can the relationship between argumentation and proof be analysed? Educational Studies in Mathematics, Netherlands, v. 66, n. 1, p. 23-41, 2007.

PERRY, P. et al. La geometría del ángulo desde otro ángulo: Una aproximación metodológica alternativa. Épsilon - Revista de Educación Matemática, España, v. 29, n. 3, p. 41-56, 2012.

PERRY, P. et al. Innovación en un aula de geometría de nivel universitario. In: SAMPER, C.; MOLINA, Ó. Geometría plana: un espacio de aprendizaje. Bogotá, Colombia: Universidad Pedagógica Nacional, 2013. p. 11-34.

PONTE, J. P. Investigar, ensinar e aprender. Actas do ProfMat 2003. Lisboa: APM, 2003. p. 25-39.

PONTE, J. P. Problemas e investigaciones en la actividad matemática de los alumnos. In: GIMÉNEZ, J.; SANTOS, L.; PONTE, J. P. La actividad matemática en el aula. Barcelona: Graó, 2004. p. 25-34.

PONTE, J. P.; FONSECA, H.; BRUNHEIRA, L. As atividades de investigação, o professor e a aulade Matemática. Actas do ProfMat 1999. Lisboa, Portugal: APM, 1999. p. 91-101.

SAMPER, C. et al. Geometría dinámica: medio para el establecimiento de condicionalidad lógica. Informe de Investigación, CIUP, UPN - COLCIENCIAS: Bogotá. 2010.

SAMPER, C.; MOLINA, O. Geometría Plana: un espacio de aprendizaje. Bogotá, Colombia: Universidad Pedagógica Nacional, 2013. 
SAMPER, C. et al. Problemas abiertos de conjeturación. In: ENCUENTRO DE GEOMETRÍA Y SUS APLICACIONES, 21., 2013b, Bogotá. Memorias... Bogotá, Colombia: Universidad Pedagógica Nacional. 2013. p. 167-170.

SAMPER, C. et al. Conjeturas y organización del contenido matemático en clase. Informe de Investigación, CIUP, UPN - COLCIENCIAS. Bogotá, Colombia: 2014.

SANTOS-TRIGO, M. Problem Solving in Mathematics. In: LERMAN, S. Encyclopedia of Mathematics Education. Dordrecht: Springer Science+Business Media, 2014. p. 496-500.

SCHOENFELD, A. H. Learning to think mathematically: Problem solving, metacognition, and sense making in mathematics. In: GROWS, D. A. Handbook of Research on Mathematics Teaching and Learning. NY: Macmillan, 1992. p. 334-370.

SHERIN, M.; RUSS, R.; COLESTOCK, A. Accessing mathematics teachers' in-the-moment noticing. In: SHERIN, M.; JACOBS, V.; PHILIPP, R. Mathematics Teacher Noticing. New York: Routledge, 2011. p. 79-94.

STEFFE, L. P.; THOMPSON, P. W. Teaching experiment methodology: Underlying principles and essential elements. In: KELLY, A. E.; LESH, R. A. Handbook of research in mathematics and science education. Mahwah, EUA: Lawrence Erlbaum Associates, 2000. p. 267-306.

STYLIANIDES, A.; STYLIANIDES, G. Content knowledge for mathematics teaching: The case of reasoning and proving. In: NOVOTNÁ, J., et al. CONFERENCE OF THE INTERNATIONAL GROUP FOR THE PSYCHOLOGY OF MATHEMATICS EDUCATION, 30., 2006, Prague. Proceedings.. Prague: PME, v. 5, 2006. p. 201-208.

STYLIANIDES, G.; STYLIANIDES, A. Validation of solutions of construction problems in dynamic geometry environments. International journal of computer for Mathematical Learning, Netherlands, v. 10, n. 1, p. 31- 47, 2005.

STYLIANIDES, J.; BALL, D. Understanding and describing mathematical knowledge for teaching: knowledge about proof for engaging students in the activity of proving. Journal of Mathematics Teacher Education, Netherlands, v. 11, n. 4, p. 307-332, 2008.

YACKEL, E.; HANNA, G. Reasoning and proof. In: KILPATRICK, J.; MARTIN, W. G.; SCHIFTER, D. A research companion to Principles and Standards for School Mathematics. Reston, VA: National Council of Teachers of Mathematics, 2003. p. 227-236.

YEVDOKIMOV, O. The place and significance of the problems for proof in learning mathematics. In: MARIOTTI, M. A. EUROPEAN RESEARCH IN MATHEMATICS EDUCATION III, 3., 2004, Bellaria. Proceedings... Bellaria: University of Pisa and ERME, 2004. Disponivel em: <http://www.mathematik.uni-dortmund.de/ erme/CERME3/Groups/T G4/TG4_Yevdokimov _cerme3.pdf>. Acesso em: Febrero de 2017

ZIMMERMANN, B. Improving of Mathematical Problem-Solving: Some New Ideas from Old Resources. In: FELMER, P.; PEHKONEN, E.; KILPATRICK, J. Posing and Solving Mathematical Problems. Switzerland: Springer International Publishing, 2016. p. 83-108.

Submetido em 13 de Janeiro de 2018. Aprovado em 25 de Agosto de 2018. 